\title{
NORMAS CONSTITUCIONALES Y LEGALES DEL CONTROL FISCAL EN EL MARCO DE LA EVALUACIÓN INTEGRAL DE LAS CONTRALORÍAS TERRITORIALES \\ EL CONTROL FISCAL
}

\begin{abstract}
CONSTITUTIONAL AND LEGAL RULES OF THE FISCAL CONTROL IN THE FRAMEWORK OF THE INTEGRAL EVALUATION OF THE TERRI-TORIALES CONTROLLERS
\end{abstract}

THE FISCAL CONTROL

\section{INTRODUCCIÓN}

Partiendo de la definición adoptada de la constitución política de 1991, El Control Fiscal es una función pública ejercida por la Contraloría General de la Republica, que sirve como herramienta para vigilar la gestión fiscal de la administración y de los particulares o entidades que manejan fondos públicos o bienes de la Nación. ${ }^{1}$

Ahora bien, con el Acto Legislativo 4 del 18 de septiembre del 2019, "Por medio del cual se reforma el régimen de control fiscal" podemos decir entre otras que lo que se busca es que la Contraloría General de la Republica a través del control fiscal preventivo y concomitante, pueda evitar los hechos de corrupción antes que estos sean consumados, es decir, que con la ejecución de dichos controles se pueda intervenir a tiempo en aras de proteger el patrimonio del Estado.

\footnotetext{
${ }^{1}$ Articulo 267 Constitución Política de Colombia/ http://wsp.presidencia.gov.co/Normativa/Documents/Constitucion-PoliticaColombia.pdf

2 "Por medio del cual se reforma el régimen de control fiscal".

${ }^{3}$ verbigracia: Corte Constitucional sentencia C-716 de 2002; C- 103 de 2015.
} 


\section{RESUMEN}

La finalidad de este documento se orienta a identificar aquellos aspectos relevantes que con la expedición del Acto Legislativo No. 04 de 2020², transforman el espectro funcional que por mandato constitucional se encuentra asignado a la Contraloría General de la Republica; para lo cual, se analizarán los pronunciamientos jurisprudenciales ${ }^{3}$, en nuestro criterio, más relevantes y en cuyo análisis se detallan los antecedentes y la justificación del desarrollo normativo que ha retratado la evolución del modelo constitucional de control fiscal en Colombia. Lo anterior, a efectos de determinar si con la expedición del acto precitado (el cual introduce el Control Fiscal Concomitante y Preventivo), se retoma el vetusto y proscrito "control previo", el cual, conllevo en su momento, una coadministración funesta y carente de responsabilidad en la que coincidían potestades de ordenación de gasto, investigación y juzgamiento, desdibujando la esencia del ejercicio de fiscalización y la autonomía e independencia que le asiste como órgano de control dentro de la estructura del estado (por virtud de los Artículos 113 y 117 de la Constitución Política).

\section{ABSTRACT}

The purpose of this document is oriented to identify those relevant aspects that with the issuance of Legislative Act No. 04 of 2020, transform the functional spectrum that by constitutional mandate is assigned to the Comptroller General of the Republic; For this, the most relevant jurisprudential pronouncements will be analyzed, in our opinion, and whose analysis details the background and justification of the regulatory development that has re-treated the evolution of the constitutional model of fiscal.centrol-in, Gelombiare, Bogotá D.c.

PBX: [571] 3186800 - 3816710 -Línea gratuita de atención ciudadana: 018000-120205

f auditoriageneral Đauditoriagen @auditoriagen $\mathbf{0}$ auditoriageneralcol

participacion@auditoria.gov.co

www.auditoria.gov.co 
The foregoing, in order to determine whether, with the issuance of the aforementioned act (which introduces the Concomitant and Preventive Fiscal Control), the old and proscribed "prior control" is resumed, which, at the time, led to a disastrous and lacking co-administration of responsibility in which powers of spending, investigation and judgment coincided, blurring the essence of the fiscalization exercise and the autonomy and independence that assists it as a control body within the state structure (by virtue of Articles 113 and 117 of the Political Constitution).

\section{PALABRAS CLAVES}

Control fiscal

Contralorías

Corrupción

Normas legales/ Normas Constitucionales

\section{WORD KEYS}

Fiscal control

Comptrollers

Corruption

Legal norms / Constitutional Norms

\section{ECUACIÓN DE BÚSQUEDA}

Control fiscal + Acto Legislativo + Normas legales/ Normas Constitucionales + Contralorías 


\section{$>$ CONTEXTO INTERNACIONAL}

Con el fin de enriquecer la búsqueda del tema "Control Fiscal", acudimos a la plataforma "Scimago Journal \& Country Rank", la cual provee una serie de indicadores sobre la calidad y el impacto de publicaciones y revistas científicas; es necesario hacer referencia en relación con los "países líderes en la investigación y producción científica del mundo" en materia de control fiscal; para lo cual, aplicando el filtro de las "Ciencias Sociales" como área del conocimiento en el marco de la OCDE y la "Administración Pública" como disciplina, es posible evidenciar los siguientes diez (10) países líderes:

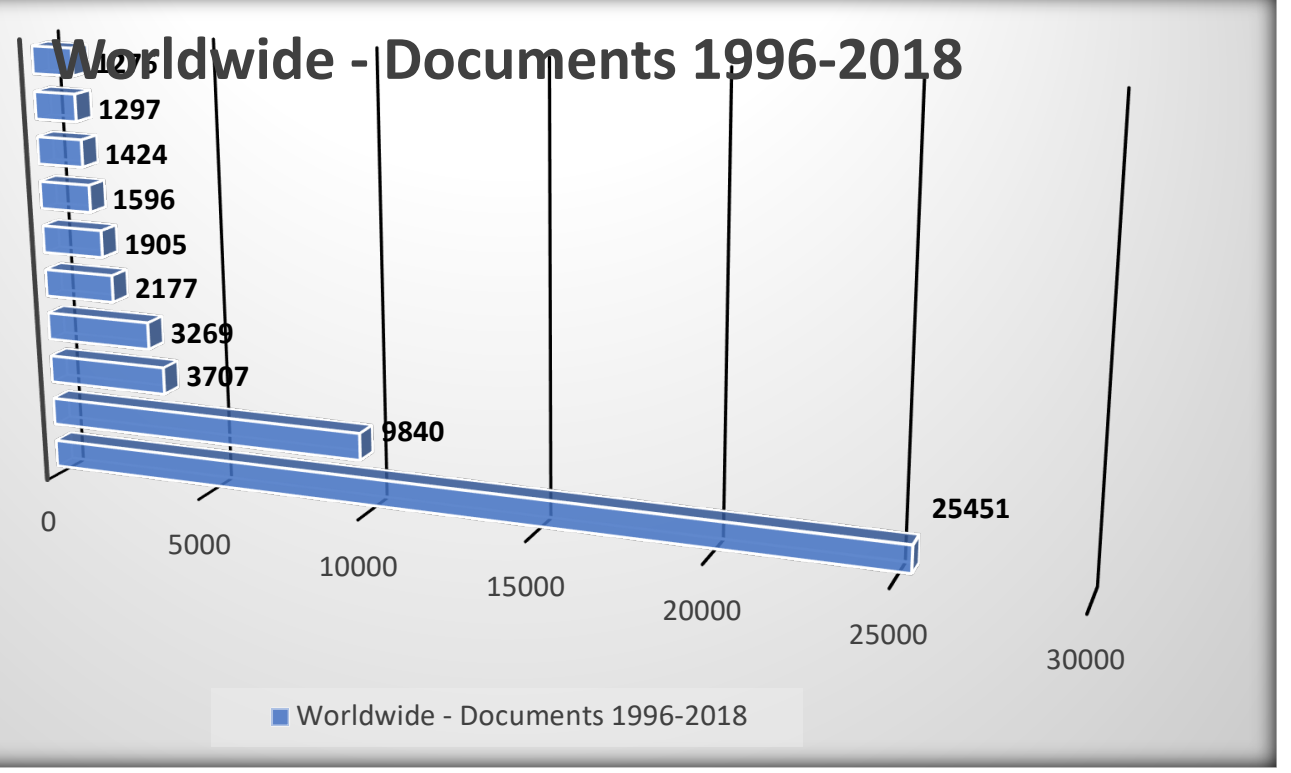

Fuente: "Scimago Journal \& Country Rank"

Cra. 57C No. 64A - 29, Barrio Modelo Norte, Bogotá D.C.

PBX: [571] 3186800 - 3816710 -Línea gratuita de atención ciudadana: 018000-120205

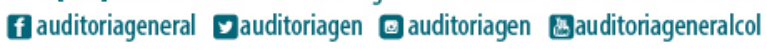

participacion@auditoria.gov.co

www.auditoria.gov.co 
Página 5 de 11

Con el ánimo de establecer el "puesto ocupa Colombia en la producción científica mundial y regional" en materia de Control Fiscal, aplicando el filtro antedicho, es posible evidenciar lo siguiente:

\section{Worldwide - Documents 1996-2018}

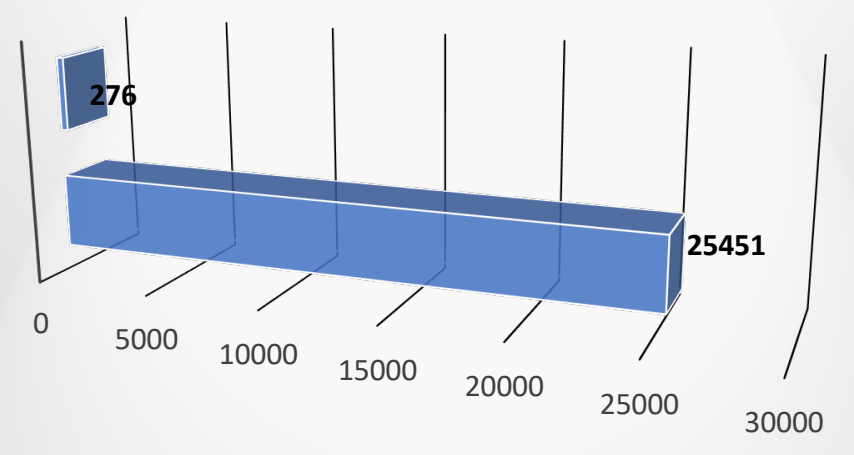

- Worldwide - Documents 1996-2018

Fuente: "Scimago Journal \& Country Rank"

\section{Regional level - 1996-2018}

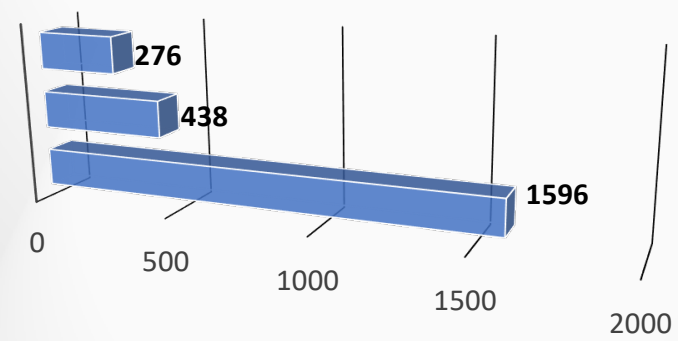

negional level - 1996-2018

Fuente: "Scimago Journal \& Country Rank"

Cra. 57C No. 64A - 29, Barrio Modelo Norte, Bogotá D.C.

PBX: [571] 3186800 - 3816710 -Línea gratuita de atención ciudadana: 018000-120205

f auditoriageneral Đauditoriagen @auditoriagen $\mathbf{0}$ auditoriageneralcol

participacion@auditoria.gov.co

www.auditoria.gov.co 
Página 6 de 11

Finalmente, frente a las "publicaciones más importantes a nivel mundial y regional" en materia de Control Fiscal, previa aplicación de los filtros antes referenciados al interior de la plataforma "Scimago Journal \& Country Rank", se pudo evidenciar lo siguiente:

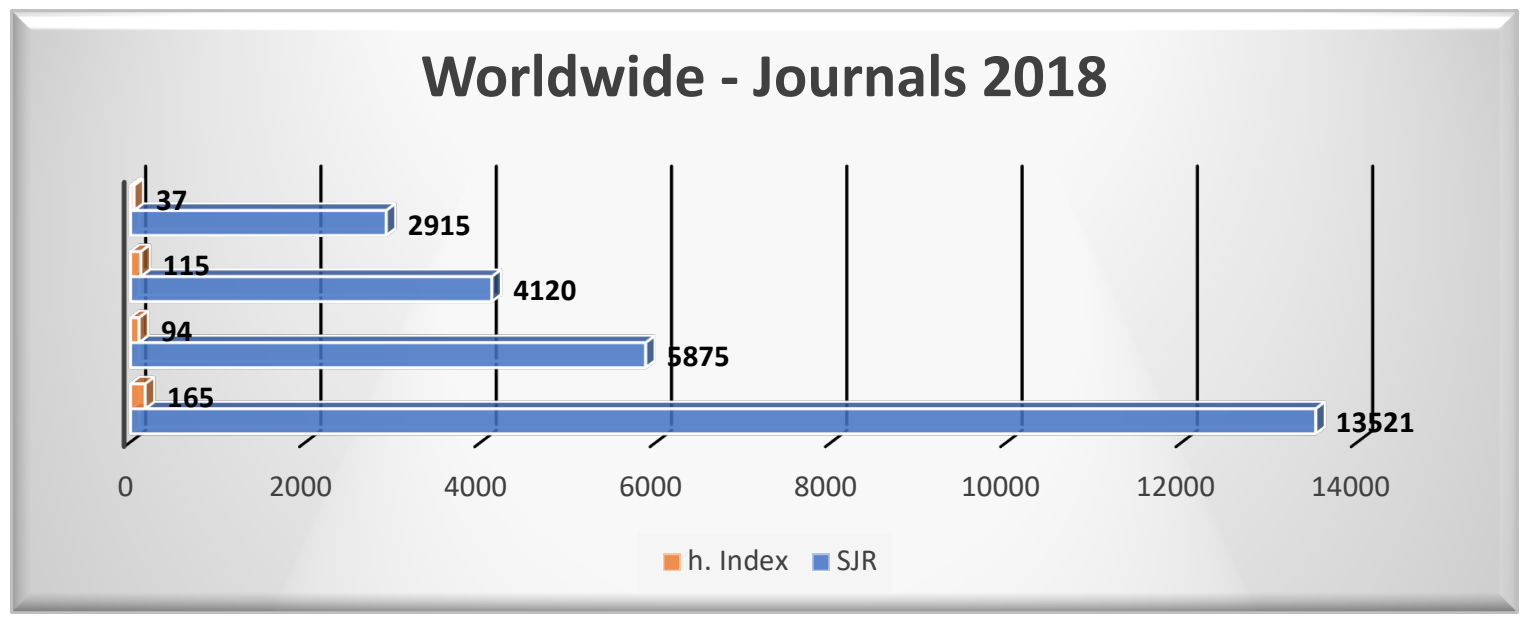

Fuente: "Scimago Journal \& Country Rank"

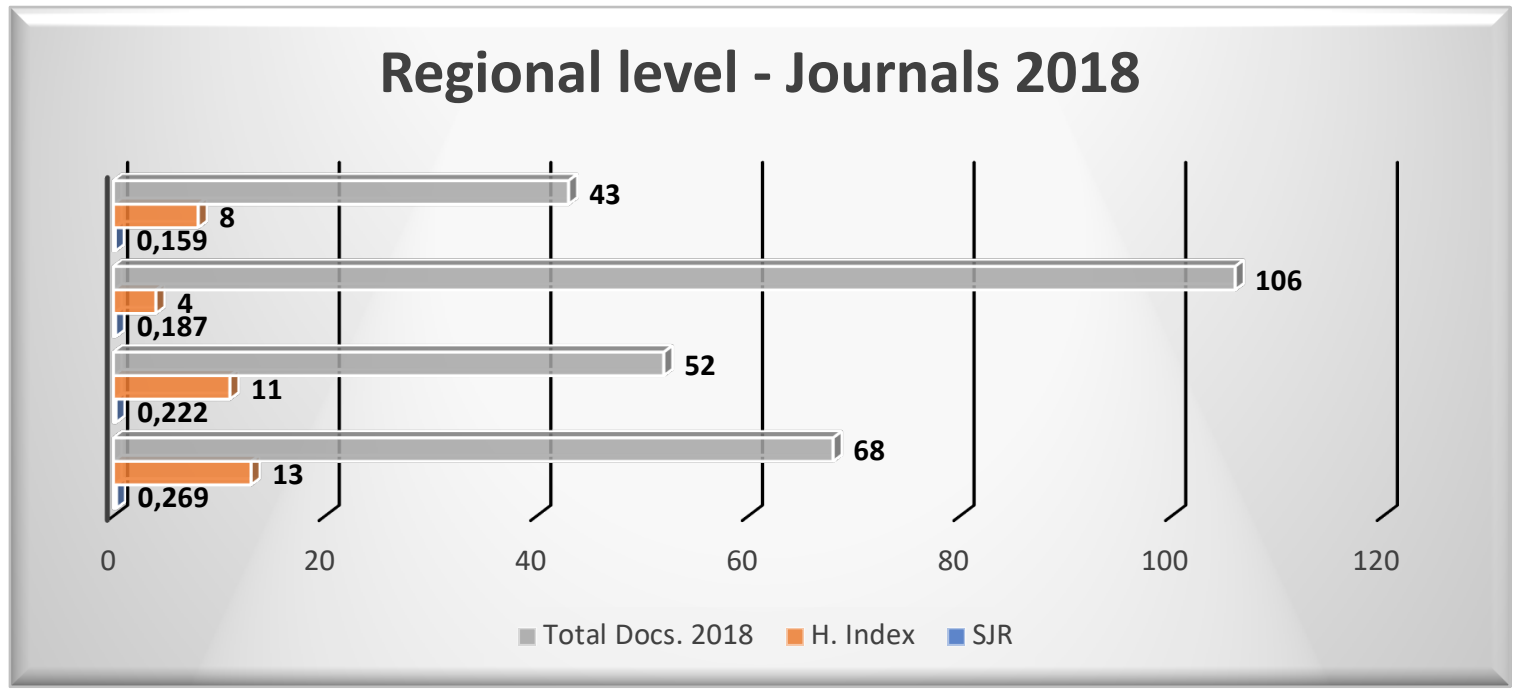

Fuente: "Scimago Journal \& Country Rank"

Cra. 57C No. 64A - 29, Barrio Modelo Norte, Bogotá D.C.

PBX: [571] 3186800 - 3816710 -Línea gratuita de atención ciudadana: 018000-120205

f auditoriageneral Đauditoriagen 巴auditoriagen $\mathbf{0}$ auditoriageneralcol

participacion@auditoria.gov.co

www.auditoria.gov.co 


\section{CONTEXTO REGIONAL}

Por otra parte, acudiendo a la Red Federada de Repositorios Institucionales de Publicaciones Científicas - La Referencia, red latinoamericana de repositorios de acceso directo, a efectos de establecer el contexto regional de las diferentes publicaciones en materia de Control Fiscal, es posible evidenciar en primera instancia que, en relación con los "países lideran la producción académica en Latinoamérica", se reportan los siguientes:

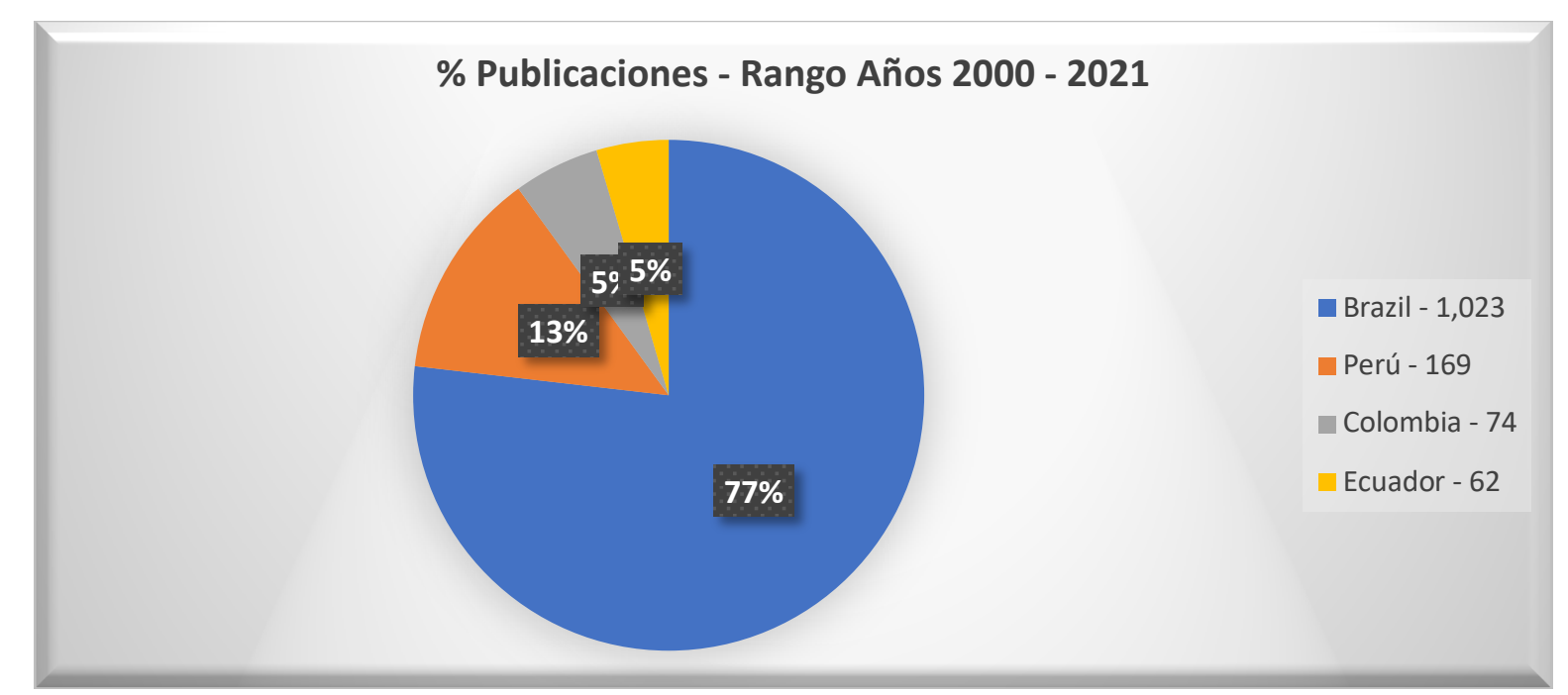

Fuente: Red Federada de Repositorios Institucionales de Publicaciones Científicas - La Referencia

En relación con los " 5 autores que más publican en Latinoamérica" en materia de Control Fiscal, es posible evidenciar lo siguiente: 


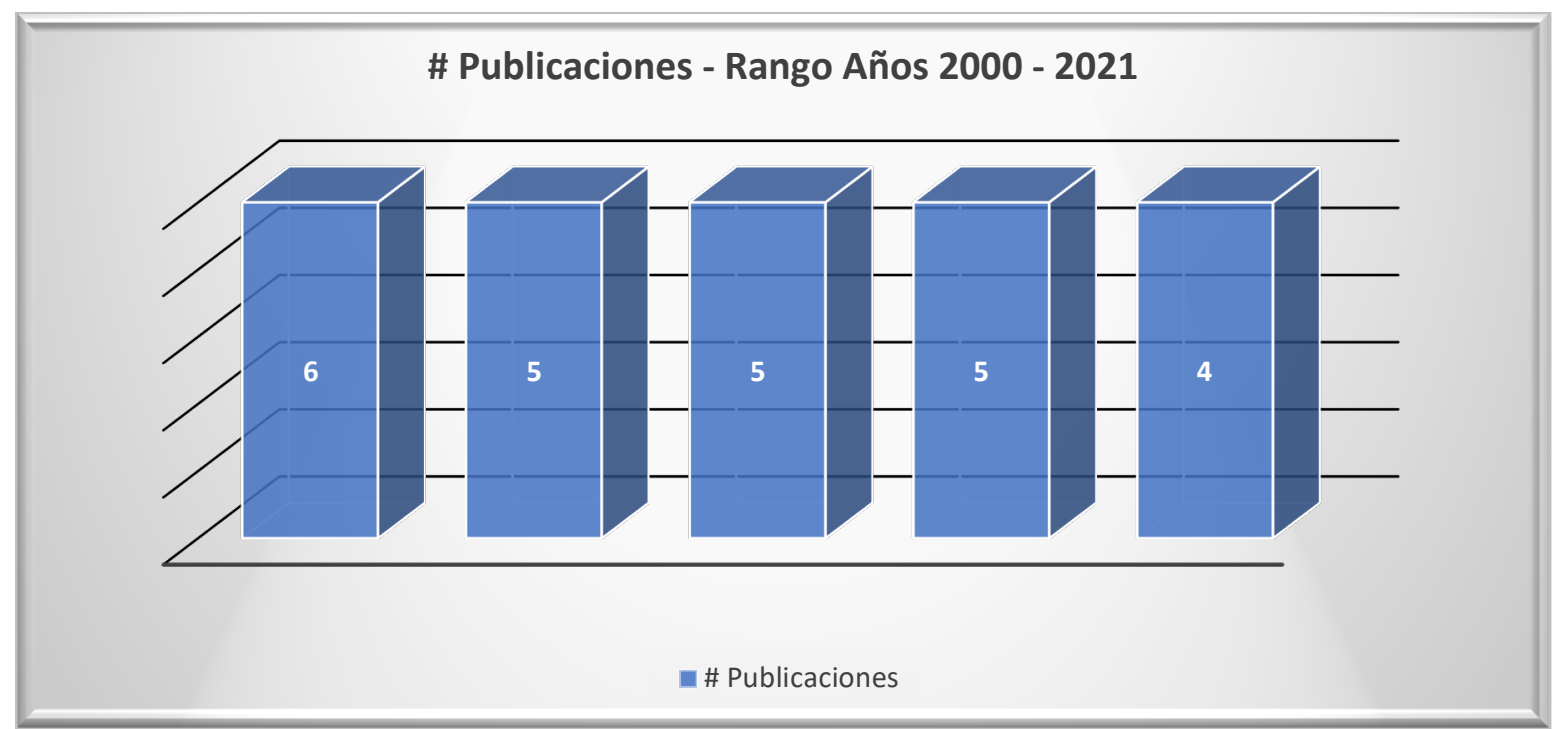

Fuente: Red Federada de Repositorios Institucionales de Publicaciones Científicas - La Referencia

En relación con las "Universidades que más publican en Latinoamérica" en materia de Control Fiscal, luego de aplicar los filtros correspondientes al momento de usar la herramienta "La República", es posible concluir lo siguiente:

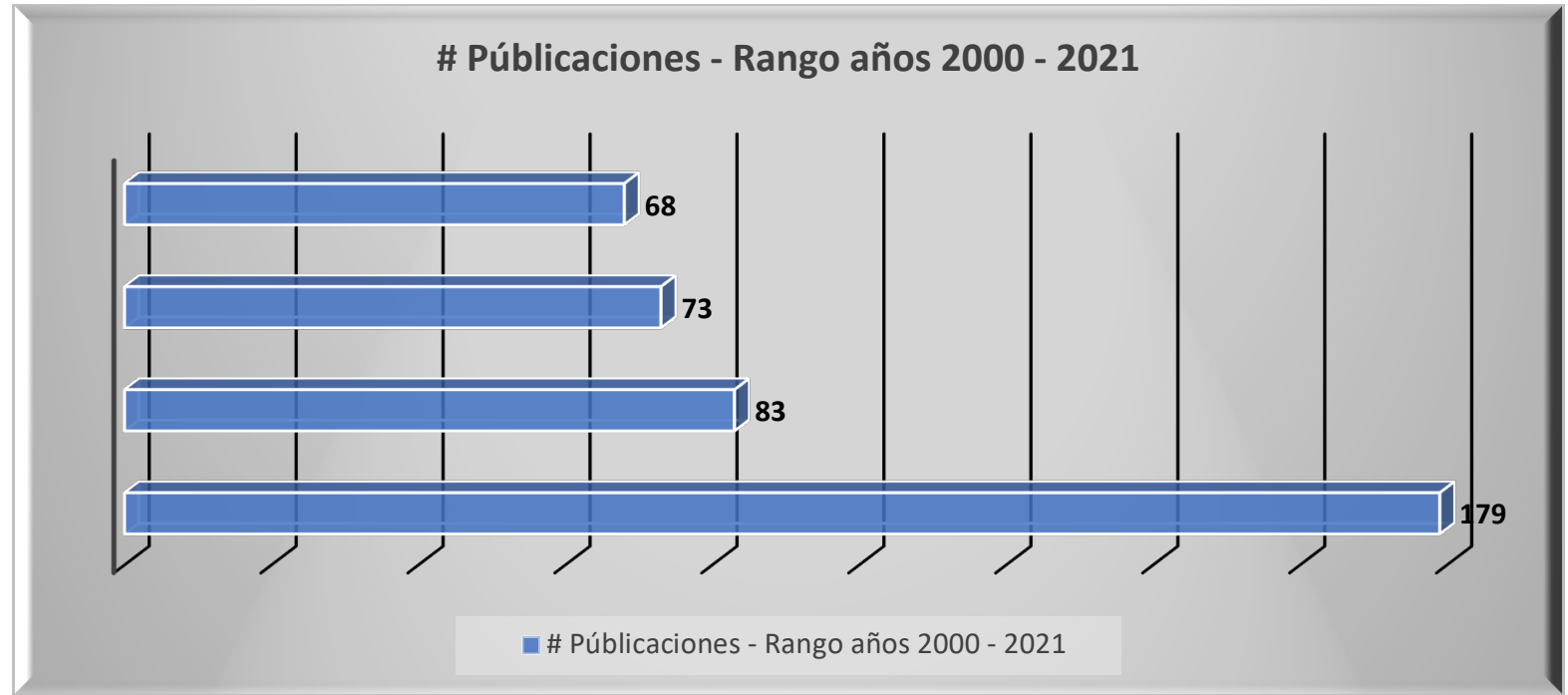

Fuente: Red Federada de Repositorios Institucionales de Publicaciones Científicas - La Referencia

Cra. 57C No. 64A - 29, Barrio Modelo Norte, Bogotá D.C.

PBX: [571] 3186800 - 3816710 -Línea gratuita de atención ciudadana: 018000-120205

f auditoriageneral Đauditoriagen @auditoriagen $\mathbf{0}$ auditoriageneralcol

participacion@auditoria.gov.co

www.auditoria.gov.co 
Finalmente, en relación con "la tipología documental de mayor publicación en Latinoamérica", cabe resaltar que, revisados el número de tipos de recurso, entre los años 2000 y 2021 , es posible encontrar que, las "tesis de maestría" se erigen como los documentos de mayor publicidad, así:

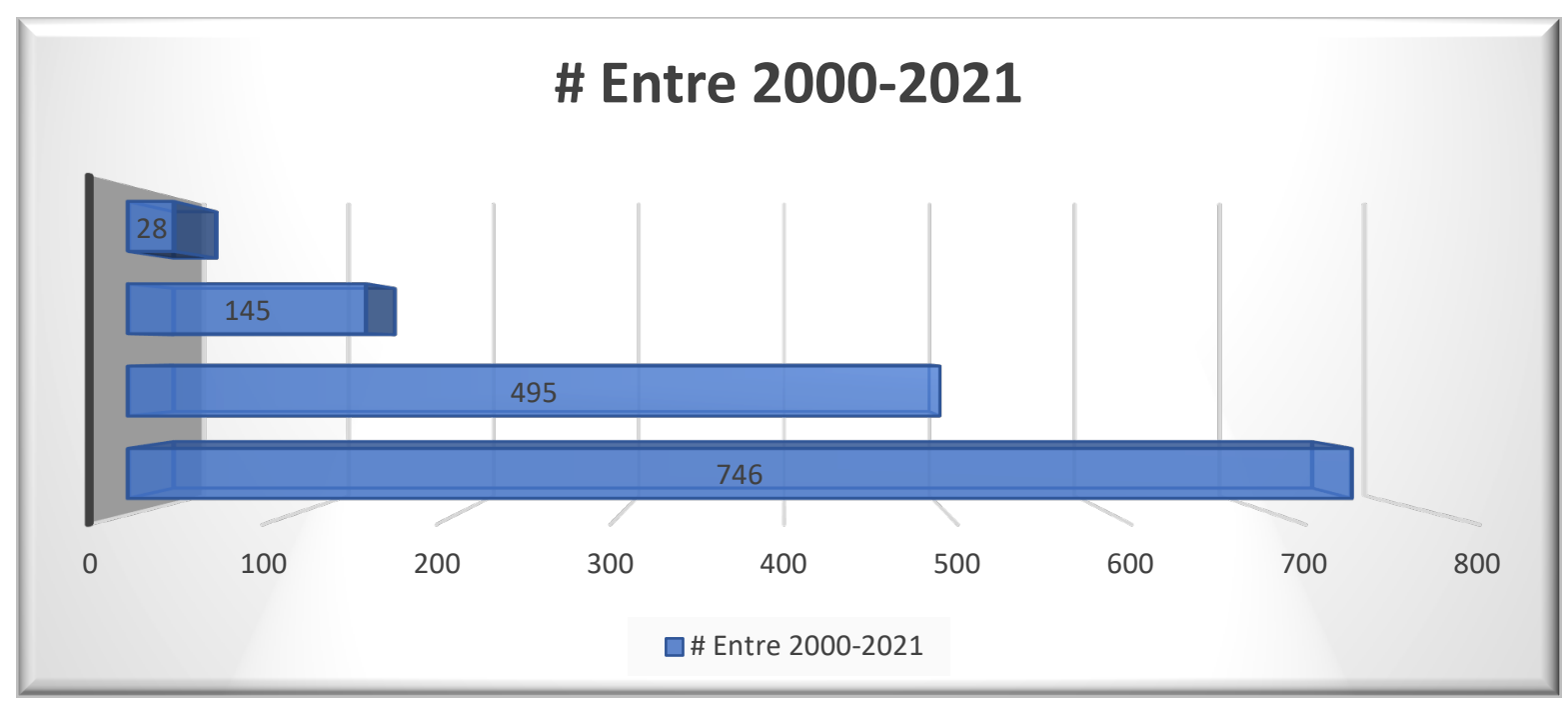

Fuente: Red Federada de Repositorios Institucionales de Publicaciones Científicas - La Referencia

\section{CONCLUSIONES}

Podemos concluir que el control fiscal es de gran importancia para el país, toda vez que lo que se persigue es frenar la lucha contra la corrupción y mal uso de los recursos públicos, aplicando para ello las normas que lo regulan. Con el Acto Legislativo 4 del 18 de septiembre del 2019, se efectuó una considerable mutación al control fiscal en Colombia, donde se incluyeron nuevas funciones a la Contraloría General de la Republica y se adoptaron nuevas modalidades de control como son el control concomitante y control preventivo, con el fin de ejercer una mayor inspección en el manejo y destinación de los recursos públicos. 
Sin embargo, a efectos de establecer el eventual retorno de pretéritas prácticas de control (previo), es necesario estructurar una línea jurisprudencial que decante a grandes rasgos las actuaciones desterradas de nuestro ordenamiento jurídico en materia de control fiscal y contrastarla con el corpus normativo que actualmente gobierna el modelo actual y la estructura de la CGR, esto es, los Decretos 403, 405, 406, 407, 408 y 409 del 16 de marzo de 2020, los cuales, han sido expedidos con ocasión al acto legislativo antes aludido y con los cuales se propende por un mayor fortalecimiento al control y vigilancia de los recursos públicos y a la transformación de las normas que lo regulan.

\section{REFERENCIAS}

- Constitución Política de Colombia

- Ley 42 de 1993

- Decreto - Ley 267 de 2000

- Ley 610 de 2000

- Ley 850 de 2003

- Ley 1474 de 2011

- Decreto 272 de 2000

- Decretos 403, 405, 406, 407, 408 y 409 del 16 de marzo de 2020.

- Corte Constitucional Sentencia C- 167 de 1995

- Corte Constitucional Sentencia C-716 de 2002

- Corte Constitucional Sentencia C- 1176 de 2004

- Corte Constitucional Sentencia C- 103 de 2015

- Acto Legislativo 04 de 2019 Congreso de la República - EVA - Función Pública. (2020). Retrieved 13 April 2020, from https://www.funcionpublica.gov.co/eva/gestornormativo/norma.php?i=100251

- 1. Principios, Fundamentos y Aspectos - Contraloría General de la República. (2020). Retrieved 13 April 2020, from https://www.contraloria.gov.co/guia-de-auditoria-en-el-marco-de-normas-issai/1.-principios-fundamentos-y-aspectos? $p \quad p$ id=110 INSTANCE 3rPGBVbCYHzC\&p $p$ lifecycle $=0 \& p$ p state $=$ normal\&p $p$ mode $=v i e w \& p$ col $i d=118$ INSTANCE gtaDd3To1WRT column$1 \& p \mathrm{p}$ col pos $=1 \& \mathrm{p} \mathrm{p}$ col count $=2 \& 110$ INSTANCE 3rPGBVbCYHzC struts action $=\% 2$

- Normatividad, Resoluciones y Conceptos - Contraloría General de la República. (2020). Retrieved 13 April 2020, from https://www.contraloria.gov.co/web/relatoria/normatividad-resoluciones-y-conceptos

- Normatividad - Contraloría General de la República. (2020). Retrieved 13 April 2020, from https://www.contraloria.gov.co/web/sireci/documentacion/normatividad d (2020). Retrieved 13 April 2020, from http://bibliotecadigital.usbcali.edu.co/bitstream/10819/4670/1/9789588785004.pdf 
Página 11 de 11

- elmundo.com: Precisiones sobre el nuevo control fiscal - Resoluciones de Nombramiento - Contraloría General de la República. (2020). Retrieved 13 April 2020, from https://www.contraloria.gov.co/contraloria/talento-humano/resolucionesde-nombramiento? $\mathrm{p}$ id $=101 \& \mathrm{p}$ p lifecycle $=0$ \& $\mathrm{p}$ state $=$ maximized\&p $p$ mode $=$ view\& 101 struts action=\%2Fasset publisher\%2Fview content\& 101 assetEntryld=1591422\& 101 type=content\& 101 urlTitle=elmundocom-precisiones-sobre-el-nuevo-control-fiscal\&inheritRedirect=false

- Actos Legislativos - Comisión Primera Constitucional Permanente. (2020). Retrieved 13 April 2020, from https://www.comisionprimerasenado.com/actolegislativo

- Pérez, D. (2020). Nuevo Régimen de Control Fiscal en Colombia, un análisis comparativo - GestioPolis. Retrieved 13 April 2020, from https://www.gestiopolis.com/nuevo-regimen-de-control-fiscal-en-colombia-un-analisis-comparativo/

- Leyes desde 1992 - Vigencia expresa y control de constitucionalidad [CONSTITUCION_POLITICA_1991]. (2020). Retrieved 13 April 2020, from http://www.secretariasenado.gov.co/senado/basedoc/constitucion politica 1991.html

- (2020). Retrieved 13 April 2020, from https://dapre.presidencia.gov.co/normativa/normativa/ACTO\%20LEGISLATIVO $\% 20$ No $\% 2004 \% 20$ DEL $\% 2018 \% 20 D E \% 20$ SEPTIEMBRE\%20DE\%202019.pdf

- (2020). Retrieved 13 April 2020, from http://wsp.presidencia.gov.co/Normativa/Documents/Constitucion-Politica-Colombia.pdf

- (2020). Retrieved 13 April 2020, from https://www.andresgarciazuccardi.com/wp-content/uploads/2018/11/“"Por-medio-del-cual-se-reforma-el-régimende-control-fiscal".pdf

- Proyecto de Acto Legislativo "Por medio del cual se reforma el régimen de control fiscal" - Andres García Zuccardi. (2020). Retrieved 13 April 2020, from https://www.andresgarciazuccardi.com/proyecto-acto-legislativo-medio-del-sereforma-regimen-control-fiscal/

- Auditoría General de la Republica, https://www.auditoria.gov.co/ 